UDC 517.9

\title{
WEXLER INEQUALITY AND ALMOST PERIODIC SOLUTIONS OF DIFFERENTIAL EQUATIONS WITH DEVIATING ARGUMENT OF MIXED TYPE
}

\section{НЕРІВНІСТЬ ВЕКСЛЕРА І МАЙЖЕ ПЕРІОДИЧНІ РОЗВ'ЯЗКИ ДИФЕРЕНЦІАЛЬНИХ РІВНЯНЬ $З$ АРГУМЕНТОМ МІШАНОГО ТИПУ, ЩО ВІДХИЛЯЕТЬСЯ}

\author{
M. U. Akhmet \\ Middle East Techn. Univ. \\ 06531 Ankara, Turkey \\ e-mail:marat@metu.edu.tr
}

\section{A. Tleubergenova}

Kazakh Nat. Techn. Univ.

463000 Aktobe, Mares'eva Str., 10

The paper is concerned with the existence and stability of an almost periodic solution of the system with deviating argument

$$
\frac{d x(t)}{d t}=A(t) x(t)+f\left(t, x(t), x\left(t-\tau_{1}(t)\right), \ldots, x\left(t-\tau_{k}(t)\right) .\right.
$$

The Wexler inequality for the Cauchy matrix is used. Conditions for stability of the solution are given.

Розглядається проблема існування та стабільності майже періодичного розв'язку системи з відхиленням в аргументі

$$
\frac{d x(t)}{d t}=A(t) x(t)+f\left(t, x(t), x\left(t-\tau_{1}(t)\right), \ldots, x\left(t-\tau_{k}(t)\right) .\right.
$$

Використовуеться нерівність Векслера. Наведено умови стабільності розв'язку.

1. Introduction and preliminaries. Different aspects of the theory of almost periodic solutions of quasilinear differential equations with deviating argument, including applications, has been investigated by many authors [1-7]. The problem of existence of almost periodic solutions for functional differential equations was considered by J. Hale [2] for the case when the non perturbed system is autonomous or periodic and the argument is retarded. The paper [4] deals with the existence of almost periodic solutions of a system with unique and constant deviation. The aim of the present paper is to investigate the problem for the system (1), where the deviations and the matrix of coefficients are almost periodic functions. Moreover, we assume that the equation is of mixed type [3], that is, the derivative of $x$ depends on the past as well as the future values of $t$. This type of equations, apparently, can be applied to problems of anticipatory systems [8]. One should emphasize that the general theory has not been considered for this type of equations as well as for the equations with retarded argument or for the systems of the neutral type $[3,9]$.

(C) M. U. Akhmet, M. A. Tleubergenova, 2004 
Let $N, R$ be sets of all natural and real numbers respectively, $\|\cdot\|$ be the Euclidean norm in $R^{n}, n \in N$. Let $s \in R$ be a positive number. We denote $G_{s}=\left\{x \in R^{n}\|x\| \leq s\right\}$ and $G_{s}^{k+1}=G_{s} \times G_{s} \times \ldots \times G_{s}$ (that is, $G_{s}^{k+1}$ is the Cartesian product of $k$ copies of $G_{s}$ ).

Let a set $C_{0}(R)$ (respectively, $C_{0}\left(R \times G_{H}^{k+1}\right)$ for a given $H \in R, H>0$ ) be a set of all functions that are bounded and uniformly continuous on $R$ (respectively on $R \times G_{H}^{k+1}$ ).

Definition 1. For $f \in C_{0}(R)$ (respectively $C_{0}\left(R \times G_{H}^{k+1}\right)$ ) and $\tau \in R$ the translation of $f$ by $\tau$ is the function $Q_{\tau} f=f(t+\tau), t \in R$ (respectively $\left.Q_{\tau} f(t, z)=f(t+\tau, z),(z, t) \in R \times G_{H}^{k+1}\right)$.

Definition 2. $A$ number $\tau \in R$ is called $\varepsilon$-translation number of a function $f \in C_{0}(R)$ $\left(C_{0}\left(R \times G_{H}^{k+1}\right)\right)$ if $\left\|Q_{\tau} f-f\right\|<\varepsilon$ for every $t \in R\left((t, z) \in R \times G_{H}^{k+1}\right)$.

Definition 3. A function $f \in C_{0}(R)\left(C_{0}\left(R \times G_{H}^{k+1}\right)\right)$ is called an almost periodic (almost periodic in $t$ uniformly with respect to $z \in G_{H}^{k+1}$ ) if for every $\varepsilon \in R, \varepsilon>0$, there exists a corresponding dense set of $\varepsilon$-translations of $f$.

Denote by $\mathcal{A P}(R)\left(\mathcal{A P}\left(R \times G_{H}^{k+1}\right)\right)$ the set of all such functions [10,11].

The aim of this paper is to investigate the problem of existence and exponential stability of an almost periodic solution of a system of differential equations with deviating argument of the form

$$
\frac{d x}{d t}=A(t) x+f\left(t, x(t), x\left(t-\tau_{1}(t)\right), \ldots, x\left(t-\tau_{k}(t)\right),\right.
$$

where $x \in R^{n}, t \in R$. We will need the following assumptions:

$\left(C_{1}\right) A(t) \in \mathcal{A} \mathcal{P}(R)$ is an $n \times n$ matrix, $\tau_{j} \in \mathcal{A P}(R), j=\overline{1, k}$.

$\left(C_{2}\right) f \in \mathcal{A P}\left(R \times G_{s}^{k+1}\right)$ for every $s \in R, s \geq 0$.

Let $X(t, s), X(s, s)=I$, be a Cauchy matrix of the associate homogeneous linear system

$$
\frac{d x}{d t}=A(t) x
$$

One of our basic assumptions is the following.

$\left(C_{3}\right) \exists\{a, b\} \subset R, b \geq 1, a>0$, such that

$$
\|X(t, s)\| \leq b \exp (-a(t-s)), t \geq s .
$$

Lemma 1 [12-14]. Let $A(t) \in \mathcal{A P}(R)$ and the condition $\left(C_{3}\right)$ be satisfied. Then

$$
\|X(t+\tau, s+\tau)-X(t, s)\|<\varepsilon \exp \left(-\frac{a}{2}(t-s)\right),
$$

if $\tau$ is $\frac{a \varepsilon}{2 b^{2}}$-translation number of $A(t)$.

Lemma 2 [10]. If $f \in \mathcal{A P}\left(R \times G_{H}^{k+1}\right)$ and $z \in \mathcal{A P}(R), z: R \rightarrow G_{H}^{k+1}$. Then $f(t, z(t)) \in$ $\in \mathcal{A P}(R)$.

Remark 1. Lemma 1 is due to D. Wexler [12] and is of principal significance in the article. It states that the matrix $X(t, s)$ is "diagonal almost periodic". Information relevant to this assertion can be found in the book by W. A. Coppel (see Proposition 4 in Lecture 8 of [15]). 
2. Main result. In this section we consider the problem of existence of an exponentially stable almost periodic solution of (1).

Lemma 3. If $\phi \in \mathcal{A P}(R)$, then $\phi\left(t-\tau_{j}(t)\right) \in \mathcal{A P}(R), j=\overline{1, k}$.

Proof. Fix $\varepsilon \in R, \varepsilon>0$. Since $\phi \in C_{0}(R)$ there exists $\delta=\delta\left(\frac{\varepsilon}{2}\right)$ such that $\left\|\phi\left(t_{1}\right)-\phi\left(t_{2}\right)\right\|<$ $<\frac{\varepsilon}{2}$ if $\left|t_{1}-t_{2}\right|<\delta$. Denote $\varepsilon_{1}=\min \left(\frac{\varepsilon}{2}, \delta\right)$. By using the method of common translation numbers $[10,11]$ one can show that there exists a corresponding dense set of $\varepsilon$-translation numbers of $\phi$ and $\tau_{j}(t)$. Let $\tau$ be one of them. Then

$$
\begin{gathered}
\left\|\phi\left(t+\tau-\tau_{j}(t+\tau)\right)-\phi\left(t-\tau_{j}(t)\right)\right\| \leq\left\|\phi\left(t+\tau-\tau_{j}(t+\tau)\right)-\phi\left(t+\tau-\tau_{j}(t)\right)\right\|+ \\
+\left\|\phi\left(t+\tau-\tau_{j}(t)\right)-\phi\left(t-\tau_{j}(t)\right)\right\|<\frac{\varepsilon}{2}+\frac{\varepsilon}{2}=\varepsilon .
\end{gathered}
$$

The lemma is proved.

Assume that the following conditions hold:

$\left(C_{4}\right) \exists l \in R, l>0$, such that

$$
\left\|f\left(t, z_{1}\right)-f\left(t, z_{2}\right)\right\| \leq l \sum_{j=0}^{k}\left\|z_{1}^{j}-z_{2}^{j}\right\|
$$

where $z_{i}=\left(z_{i}^{0}, \ldots, z_{i}^{k}\right) \in R^{n(k+1)}, i=1,2$.

Fix $\sigma \in R, 0<\sigma<a$. Denote

$$
\tau_{0}=\max \left\{0, \sup _{t} \tau_{j}(t), j=\overline{1, k}\right\}, \quad m(l)=1-\frac{\exp \left(\sigma \tau_{0}\right) b l k}{a-\sigma}
$$

We assume in addition that $l$ is sufficiently small so that

$$
\begin{aligned}
& \left(C_{5}\right) l<\frac{b k}{a} ; \\
& \left(C_{6}\right) m(l)>0 .
\end{aligned}
$$

Theorem 1. Assume that $\left(C_{1}\right)-\left(C_{5}\right)$ hold. Then there exists a unique almost periodic solution $\xi_{0}(t)$ of $(1)$.

Proof. Let $T$ be an operator on $\mathcal{A P}(R)$ such that

$$
T \phi=\int_{\infty}^{t} X(t, s) F_{\phi}(s) d s
$$

Let $\phi \in \mathcal{A P}(R)$.

By using Lemma 3 one can prove that $F_{\phi}(t) \in \mathcal{A P}(R)$, and by Lemmas 1,2 on the base of the method of common translation numbers one can verify that there is a corresponding dense 
set $\Gamma \subset R$ of $\varepsilon$-translation numbers for $F_{\phi}$ such that if $\tau \in \Gamma$ then (4) is valid for $t \geq s$. Then

$$
\begin{gathered}
\|T \phi(t+\tau)-T \phi(t)\|=\left\|\int_{\infty}^{t} X(t+\tau, s) F_{\phi}(s) d s-\int_{\infty}^{t} X(t, s) F_{\phi}(s) d s\right\| \leq \\
\leq \int_{\infty}^{t}\|X(t+\tau, s+\tau)\|\left\|F_{\phi}(s+\tau)-F_{\phi}(s)\right\| d s+ \\
\quad+\int_{\infty}^{t}\|X(t+\tau, s+\tau)-X(t, s)\|\left\|F_{\phi}(s)\right\| d s \leq \\
\leq \int_{\infty}^{t} b \exp (-a(t-s)) \varepsilon d s+\int_{\infty}^{t} \varepsilon \exp \left(-a \frac{a}{2}(t-s)\right) M_{\phi} d s=\frac{b+2 M_{\phi}}{a} \varepsilon
\end{gathered}
$$

where $M_{\phi}=\sup _{t}\left\|F_{\phi}\right\|$. Thus $T \phi \in \mathcal{A P}(R)$. Moreover if $\phi_{1}, \phi_{2} \in \mathcal{A} \mathcal{P}(R)$ then, for every $t \geq t_{0}$

$$
\begin{aligned}
\left\|T \phi_{1}(t)-T \phi_{2}(t)\right\| & =\left\|\int_{-\infty}^{t} X(t, s) F_{\phi_{1}}(s) d s-\int_{-\infty}^{t} X(t, s) F_{\phi_{2}}(s) d s\right\| \leq \\
& \leq \int_{-\infty}^{t}\|X(t, s)\|\left\|F_{\phi_{1}}(s)-F_{\phi_{2}}(s)\right\| d s \leq \\
& \leq \int_{-\infty}^{t} b \exp (-a(t-s)) l k\left\|\phi_{1}-\phi_{2}\right\|_{0} d s=\frac{b k l}{a}\left\|\phi_{1}-\phi_{2}\right\|_{0} .
\end{aligned}
$$

The last inequality implies that

$$
\left\|T \phi_{1}(t)-T \phi_{2}(t)\right\|_{0} \leq \frac{b k l}{a}\left\|\phi_{1}-\phi_{2}\right\|_{0}
$$

and the condition $\left(C_{5}\right)$ implies that the operator $T: \mathcal{A P}(R) \rightarrow \mathcal{A P}(R)$ is contractive. Thus there exists a unique fixed point $\xi_{0} \in \mathcal{A P}(R)$ of $T$ which is a solution of (1).

The theorem is proved.

Remark 2. Apparently, the general problem of existence of solutions for equations of mixed type has not been considered yet. Even for the case of advanced argument there are certain difficulties if we try to define a solution for inceasing $t[16,17]$. J. Hale remarked in [3] that "these equations seem to dictate that boundary conditions should be specified in order to obtain a solution in the way as one does for elliptic partial differential equations". We regard the 
boundedness of the solution on $R$ as a boundary condition in the proof of Theorem 1 . Authors of [18] used this method to prove existence of a bounded solution for the equation of advanced type when $t \geq t_{0}$.

Formulation of stability conditions for the solution $\xi_{0}(t)$. Fix $t_{0} \in R$, denote $C\left[t_{0}-\tau_{0}, t_{0}\right]$ the set of all initial functions. Let $x(t)$ be a solution of (1) such that

$$
\left(D_{1}\right) x(t)=\left\{\begin{array}{lll}
\pi(t) \in C\left[t_{0}-\tau_{0}, t_{0}\right], t_{0}-\tau_{0} \leq t \leq t_{0}, & \text { if } & \tau_{0}>0, \\
x_{0} \in R^{n}, & \text { if } & \tau_{0}=0 .
\end{array}\right.
$$

$\left(D_{2}\right) x(t)$ satisfies the equation (1) for all $t \geq t_{0}$.

Definition 4. The solution $\xi_{0}(t)$ is called uniformly exponential stable if there exists a number $\alpha \in R, \alpha>0$, such that for every $\varepsilon>0$ there exists a number $\delta=\delta(\varepsilon)$ such that the inequality $\max _{t_{0}-\tau_{0} \leq t \leq t_{0}}\left\|\pi(t)-\xi_{0}(t)\right\|<\delta$ (or inequality $\left\|x_{0}-\phi\left(t_{0}\right)\right\|<\delta$, if $\tau_{0}=0$ ) implies that there exists a unique solution $x(t)$ which satisfies conditions $\left(D_{1}\right),\left(D_{2}\right)$ and $\left\|x(t)-\xi_{0}(t)\right\|<$ $<\varepsilon \exp \left(-\alpha\left(t-t_{0}\right)\right)$ for all $t \geq t_{0}$.

Remark 3. Analyzing definitions of Lyapunov stability for different types of equations it is possible to stress the following two generic conditions. The first one is the closeness of the initial values (the initial functions) for a solution whose stability is tested and a neighbour solution. The second one is the condition that the process after the initial moment of time should be governed only by a differential equation. These circumstances are described by conditions $\left(D_{1}\right)$ and $\left(D_{2}\right)$. And evidently, $\left(D_{2}\right)$ induces the condition of ignoring the negative values of $\tau_{j}(t)$ for the construction of initial conditions. We decided to make this comment after Definition 4, since the formulation of stability of solutions of the equations with mixed type of deviating arguments has not been encountered in the literature previosly.

The following theorem holds.

Theorem 2. Assume that $\left(C_{1}\right)-\left(C_{6}\right)$ hold. Then the almost periodic solution $\xi_{0}(t)$ of $(1)$ is uniformly exponential stable.

Proof. One can see that $v(t)=x(t)-\xi_{0}(t)$ is a solution of the equation

$$
\frac{d v}{d t}=A(t) v+w\left(t, v(t), v\left(t-\tau_{1}(t)\right), \ldots, v\left(t-\tau_{k}(t)\right)\right.
$$

where

$$
\begin{gathered}
v(t)=\theta(t)\left(\theta=\pi(t)-\xi_{0}(t)\right) \quad \text { on } \quad\left[t_{0}-\tau_{0}, t_{0}\right], \\
w\left(t, v(t), v\left(t-\tau_{1}(t)\right), \ldots, v\left(t-\tau_{k}(t)\right)=\right. \\
=f\left(t, \xi_{0}(t)+v(t), \ldots, \xi_{0}\left(t-\tau_{k}(t)\right)+v\left(t-\tau_{k}(t)\right)\right)-f\left(t, \xi_{0}(t), \ldots, \xi_{0}\left(t-\tau_{k}(t)\right),\right.
\end{gathered}
$$

and $w$ satisfies

$$
\left\|w\left(t, v_{1}\right)-w\left(t, v_{2}\right)\right\| \leq l \sum_{j=0}^{k}\left\|v_{1}^{j}-v_{2}^{j}\right\|
$$


$v_{i}=\left(v_{i}^{0}, \ldots, v_{i}^{k}\right) \in R^{n(k+1)}, i=1,2$. Thus we can reduce the problem of stability of $\xi_{0}(t)$ to the problem of stability of the zero solution $v=0$ of (9). Let us fix $\varepsilon>0$ and denote $K(l, \delta)=\frac{b}{m(l)} \delta$, where $\delta \in R, \delta>0$. Take $\delta$ so small that $K(l, \delta)<\varepsilon$. Assume, without lost of any generality, that $t_{0}=0, \tau_{0}>0$ and let $C\left[-\tau_{0}, 0\right]$ be the set of all initial functions. Fix $\theta(t) \in C\left[-\tau_{0}, 0\right]$ such that $\max _{\left[-\tau_{0}, 0\right]}\|\theta(t)\|<\delta$ and let

$$
\Psi_{\theta}=\left\{\phi \in C_{0}\left[-\tau_{0},+\infty\right) \mid \phi(t)=\theta(t) \quad \text { if } \quad-\tau_{0} \leq t \leq 0,\|\phi(t)\|_{1} \leq K(l, \delta) \exp (-\sigma t)\right\},
$$

where $C_{0}\left[-\tau_{0},+\infty\right)$ denotes the restrictions of all functions from $C_{0}(R)$ to $\left[-\tau_{0},+\infty\right)$ and $\|\phi\|_{1}=\sup _{t \geq t_{0}}\|\phi(t)\|$. Define on $\Psi_{\theta}$ an operator $\Pi$ (see [2]) such that if $\phi \in \Psi_{\theta}$ then

$$
\Pi \phi=\left\{\begin{array}{cl}
\theta(t) & \text { for all } \quad-\tau_{0} \leq t \leq 0, \\
X(t, 0) \theta(0)+\int_{0}^{t} X(t, s) F_{\phi}(s) d s, & \text { otherwise } .
\end{array}\right.
$$

We shall show that $\Pi: \Psi_{\theta} \rightarrow \Psi_{\theta}$. Indeed, for $t \geq 0$ we have that

$$
\begin{aligned}
\|\Pi \phi\| & \leq b \exp (-a t) \delta+\int_{0}^{t} b \exp (-a(t-s)) l K(l, \delta) \sum_{j=0}^{k+1} \exp \left(-\sigma\left(s-\tau_{j}(s)\right)\right) d s \leq \\
& \leq \exp (-\sigma t)\left[b \delta+\frac{(k+1) \exp \left(\sigma \tau_{0}\right) b l K(l, \delta)}{a-\sigma}\right]=K(l, \delta) \exp (-\sigma t) .
\end{aligned}
$$

Differentiating $\Pi \phi$ on $[0, \infty)$ it is easy to show that $[\Pi \phi]^{\prime}$ is a function uniformly bounded on $[0, \infty)$ and, hence, $\Pi \phi$ is a uniformly continuous function.

Let $\phi_{1}, \phi_{2} \in \Psi_{\theta}$. Then

$$
\left\|\Pi \phi_{1}-\Pi \phi_{2}\right\| \leq \int_{0}^{t} b \exp (-a(t-s)) l k\left\|\phi_{1}-\phi_{2}\right\|_{1} d s \leq \frac{b l k}{a}\left\|\phi_{1}-\phi_{2}\right\|_{1} .
$$

From $\left(C_{5}\right)$ it follows that there is a unique fixed point of the operator $\Pi: \Psi_{\theta} \rightarrow \Psi_{\theta}$ which is a solution $v(t)$ of (9) such that $x(t)=v(t)+\xi_{0}(t)$ is a unique solution of (1) which satisfies conditions $\left(D_{1}\right),\left(D_{2}\right)$.

The theorem is proved.

1. Gopalsamy K. On an almost periodic delay-differential system with almost periodic delays // Math. Jap. 1985. - 30. - P. 849-856.

2. Hale J. Theory of functional differential equations. - New York etc.: Springer, 1977.

3. Hale J. Functional differential equations. - New York etc.: Springer, 1971.

4. Lin Qing Cong. Almost periodic solutions of a class of higher-dimensional almost periodic systems with deviating arguments (Chinese. English, Chinese summary) // J. Zhangzhou Teach. Coll. Nat. Sci. - 1999. 12. - P. $21-24$. 
5. Seifert G. Almost periodic solutions for undamped nonhomogeneous delay-differential equations // Proc. Amer. Math. Soc. - 2002. - 130. - P. 2001-2005 (electronic).

6. Xu Jian Hua, Wang Zhi Cheng. Almost periodic solutions of a class of almost periodic predator-prey systems with time delay // Acta math. appl. sinica. - 2000. - 23. - P. $394-402$.

7. Zhang Shunian, Zheng Guang. Almost periodic solutions of delay difference systems // Appl. Math. Comput. - 2002. - 131. - P. $497-516$.

8. Corduneanu C. Some remarks on functional equations with advanced-delayed operations // Sixth Int. Conf. Comput. Anticipatory Systems, HEC Liege, Belgium (August 11-16, 2003): Abstract book / Ed. D. M. Dubois. - P. 4.

9. Driver R. D. Ordinary and delay differential equations // Appl. Math. Sci. - New York, Heidelberg: Springer, 1977. -20.

10. Corduneanu C. Almost periodic functions. - New York etc.: Int. Publ., 1968.

11. Fink A. M. Almost periodic differential quations // Lect. Notes Math. - Berlin etc.: Springer, 1974.

12. Wexler $D$. Solutions périodiques et presque-périodiques des systémes d'équations différetielles aux impulsions // Rev. roum. math. pures et appl. - 1965. - 10. - P. 1163-1199.

13. Halanay A., Wexler D. Teoria calitativă a sistemelor cu impulsuri. - Bucuresti: Editura Acad. Republ. Soc. România, 1968.

14. Samoilenko A. M., Perestyuk N. A. Impulsive differential equations. - Singapore: World Sci., 1995.

15. Coppel W. A. Dichotomies in stability theory. - Berlin etc.: Springer, 1978.

16. El'sgol'ts L. E. Introduction to the theory of differential equations with deviating arguments. - San Francisco etc.: Holden-Day Inc., 1966.

17. Zverkin A. M., Kamenskii G. A., and Norkin S. B. On the formulation of initial conditions for differential equations with advanced argument (Russian) // Uspekhi Mat. Nauk. - 1960. - 15. - P. $133-136$.

18. Doss $S$., Nasr S. K. On the functional equation $y^{\prime}=f(x, y(x), y(x+h)), h>0 / /$ Amer. J. Math. -1953 . 75. - P. $713-716$.

Received 28.05.2004 